\title{
Leader-Follower Equilibria for Power Markets in Presence of Prosumers
}

\author{
Sepehr Ramyar \\ Baskin School of Engineering \\ University of California, Santa Cruz \\ sramyar@ucsc.edu
}

\author{
Yihsu Chen \\ Baskin School of Engineering \\ University of California, Santa Cruz \\ yihsuchen@ucsc.edu
}

\begin{abstract}
Increased penetration of distributed energy resources throughout the power sector has introduced a new entity in electricity markets, namely, prosumers, with the dual nature of concurrent consumption and generation. This paper assesses the market power potential of prosumers (leader) using a Stackelberg model formulated as a mathematical program with equilibrium constraints (MPEC). The MPEC is recast to a mixed integer program where the Wolfe's duality is used to overcome the bilinear terms in the objective function, and disjunctive constraints are used to replace complementarity conditions. Our case study shows that the Stackelberg strategy always yields higher payoff for the prosumers compared to Cournot and perfect competition cases. Moreover, the social surplus resulted from Stackelberg equilibrium, compared to the other strategies, is the highest (lowest) when the prosumer is in short (long) position. Our analysis contributes to understanding the potential outcomes when prosumers are introduced to marketplace in the power sector.
\end{abstract}

\section{Introduction}

The power sector is going through rapid transformations in terms of available technologies and architecture. Driven by a need for decarbonization, sustainability, and resilience, we have witnessed a remarkable move towards advancing and deploying distributed renewable as well as price-responsive energy management systems. These include both demand response and storage, which altogether form the broader concept of distributed energy resources (DERs). This paradigm shift towards a more engaged demand-side challenges the conventional, top-down power grid architecture based on supply-side and calls for a new market design for the power sector. Aggregators, that facilitate the interaction between prosumers and the wholesale market by collecting and integrating DERs, would play an increasingly important role in the reliability and efficiency of power markets by offering aggregated energy bundles. These energy products are procured from a wide set of geographically and technologically different sources, rom rooftop solar panels to electric vehicle charging and smart energy management systems. This, consequently, emphasizes the significance distribution-level operations of the power market with emerging prosumers [1]. In particular, as these prosumers, with the ability of concurrent generation and consumption, are introduced to the power sector, it is expected to trigger changes in economic incentives, which might create opportunities for manipulations, thereby undermining efficiency of the power market.

To align incentives with the desired outcomes of the power market, it is imperative to assess how might the new entity, prosumers, impact market outcomes given current market rules. Game-theoretical models have extensively been used to evaluate electricity market outcomes, see for example [2] and [3]. The strength of the approach is that it allows representing the interactions among different market participants, especially new ones, while considering market rules and other institutional settings.

A recent thread of literature has also been focused on the role of aggregators as middle-men in the power sector that operate DERs on behalf of owners over a wide and diverse set of geographical area and form a considerable capacity in terms of DERs as bundled and offered into the market [4], [5]. The heterogeneity in terms of geographical placement and type of resources empowers the prosumers in the market, as the information is likely to be private only known by prosumers. This also calls for a need for examining DER's market power potential in the market as in [6]. Other studies have shown that even low levels of wind penetration could enable exercise of market power, leading to welfare loss [7].

Several studies have addressed power market outcomes in presence of this asymmetrical information situation for aggregators. For instance, [8] examines 
the impact of a DR aggregator operating a green energy management system on the wholesale market by implementing a quantity-based (Cournot) strategy. The paper, however, i) does not reflect the dual nature of a prosumer in terms of concurrent generation and consumption, and ii) is limited to Cournot strategy by the aggregator, which is just one of the several strategies at the aggregator's disposal. In a more recent work, [9], a game in the electricity market is modeled in a Stackelberg setting where the aggregator is the leader, and the grid operator along with other producers are the followers. The paper finds that the aggregator who operates a fleet of renewable generation units is able to exercise significant market power by curtailment of its power output. Here, as in [8], the aggregator is basically modeled as a supplier and is unable to reflect the buyer's power a prosumer can demonstrate. Paper [10] is closely related to ours. The paper examines the economic efficiency loss associated with aggregator, a leader, who acts as intermediate entity that facilitaes prosumers' access to the bulk market. Numerical example illustrates improved efficiency in the market brought in by DER participation is offset by the for-profit aggregator. Therefore, while leader-follower models, such as [9] [10], can provide useful insight into how prosumers can exercise market power, a Stackelberg formulation that explicitly takes the dual nature of prosumers into account and endogenizes power price formation is therefore of particular interest. The Stackelberg game has long been used to model sequential move games or leader-follower situations [11]. Examples of Stackelberg model applications in the energy sector include $[12,13,14,15,16,17,18]$.

In this paper, we build on previous work [19] to examine prosumers' market power potential. Particularly, we formulate the power market in a Stackelberg setting where the prosumer is the leader with other agents as followers. In this case, the prosumer has an information advantage and is able to internalize the best responses of other market participants into its own profit maximization problem. The optimization problem of the prosumer constitutes a mathematical program with equilibrium constraints or MPEC, which is difficult to solve due to its non-convexity in feasible set. Moreover, the resulting objective function contains bilinear terms, including the product of dual and primal variables, thereby posing numerical challenges. We overcome this difficulty by using Wolfe's duality and disjunctive representation of complementarity conditions and recast the problem into a mixed integer quadratic program.

When applying the model to the IEEE RTS 24-bus system, the numerical results indicate that the prosumer is able to do better economically, even only marginally, than price-taking and Cournot strategies in a Stackelberg equilibrium regardless of the levels of renewable capacity (or output). For other market participants and outcomes, the result of the Stackelberg strategy is in-between those of Cournot and perfect competition, thereby consistent with economic theory. The impact on social surplus under the Stackelebrg equilibrium, however, is ambiguous, depending on the prosumer's position in equilibrium. In the short position, the Stackelberg equilibrium yields the highest social surplus with the prosumer acting as an informed consumer; while in the long position, the social surplus resulting from Stackelberg equilibrium is the lowest compared to Cournot and perfect competition as the prosumer behaves as a producer with more leverage.

\section{Model Setup}

\subsection{Mathematical Programs with Equilibrium Constraints (MPEC)}

Mathematical Programs with Equilibrium Constraints are a class of optimization problems where the constraint set is in a complementarity form. Formally, according to [20], an MPEC is formulated as:

$$
\begin{aligned}
& \text { minimize } f(x, y) \\
& \text { subject to } \\
& (x, y) \in Z, \\
& y \in \mathcal{S}(x)
\end{aligned}
$$

where $x \in R^{n}, y \in R^{m}, f: R^{m+n} \rightarrow R$, $F: R^{m+n} \rightarrow R^{m}$, and $Z \subseteq R^{m+n}$. We also define a set-valued mapping $C: R^{n} \rightarrow R^{m}$ that is a closed convex subset of $R^{m}$ for each $x \in R^{n}$. Letting $X$ be the projection of $Z$ onto $R^{n}$, for all $x \in X$, $\mathcal{S}(x)$ is the solution of variational inequality defined as $(v-y)^{\top} F(x, y) \geq 0, \quad \forall v \in C(x)$.

In other words, an MPEC is an optimization problem that includes equilibrium or complementarity constraints (the variational inequality $y \in \mathcal{S}(x)$ ). One can also think of an MPEC as optimization problem faced by a leader (upper level problem) whose actions impact the equilibrium of a market (lower level problem) which, in turn, impacts the objective of the leader. The next section discusses this in detail.

\subsection{Upper and Lower Level Market Players}

Here, we introduce the upper and lower level optimization problems in the market, including 
consumers, prosumers, producers, and the grid operator. In what follows, we first introduce the notations that are used throughout the paper and then explain how each agent participates in the market.

Let $I$ denote the set of nodes (or locations) and $K$ be the set of transmission lines consisting of elements in ordered pairs of distinct nodes. $F$ is the set of generation firms, and $H$ is set of generation units hence $H_{f i} \in H$ is the set of generation units owned by firm $f$ at node $i$. We also introduce $\Omega$ and $\Delta$ as the set of primal and dual variables, respectively. Finally, we note that the Greek variables within the parenthesis to the right of an equation render the corresponding dual variable.

In this study, the prosumer is the modeled as the leader in a Stackelberg game. The prosumer at node $i$ is assumed to possess some renewable capacity with a negligible short-run marginal cost. ${ }^{1}$ The output from renewable is denoted by $K_{i}$, which is uncertain because it is dependent on available natural resources, e.g., solar and wind. Meanwhile, the prosumer also owns a dispatchable or backup resource with a capacity of $G_{i}$ in order to hedge against uncertain output $K_{i}$.

2.2.1. Upper-Level Problem For the purposes of this study, the prosumer's benefit function of consuming electricity is given by $B_{i}\left(l_{i}\right)$, where $l_{i}$ corresponds to the quantity consumed by prosumer when renewable output equals $K_{i}{ }^{2}$. Since the prosumer owns zero marginal cost renewable with an expected output of $K_{i}$, the function $B_{i}$ is formulated as "forgone" benefit of consumption if its consumption is less than $K_{i}$. The benefit function $B(\cdot)$ is assumed to be increasing and strictly concave. The monotonicity of $B(\cdot)$ indicates that the prosumer's objective function is increasing in the level of consumption. We posit that a prosumer maximizes its profit by deciding on i) amount of power to buy from $\left(z_{f i}<0\right)$ or sell to $\left(z_{f i}>0\right)$ firm $f$ in node $i$ through bilateral contracts ${ }^{3}$, ii) amount of foregone consumption, $K_{i}-l_{i}$, and iii) amount of power to be generated from the backup dispatchable technology, $g_{i}$. We also assume that the prosumer is only allowed to

\footnotetext{
${ }^{1}$ Individual "behind-the-meter" prosumers, e.g., owner of a roof-top solar panel, might have limited access to the wholesale or bulk market and be subject to fixed tariff when selling their surplus power back to the grid. We assume that the prosumer (or a aggregator) that we present here is a result of the aggregation of a large number of prosumers, thereby allowing to interact with the bulk market directly.

${ }^{2} B_{i}$ is entirely separate and different from $p_{i}\left(d_{i}\right)$, which represents willingness-to-pay or benefit of consumers in the wholesale market. It represents a local benefit function centered around consumption level at $K_{i}$. As a prosumer engages in the market, directly through bilateral trading with firms, there is limited opportunity for the market to solicit prosumers' preferences through market settlements, i.e., a preference revelation process.

${ }^{3}$ Because the equivalence between a power market based on pool-type transactions and on bi-lateral contracts have been alluded to in [3], we believe that our assumption herein is reasonable and can be seen as an extension.
}

sell/buy from node $i$ [21]. The optimization problem faced by the prosumer at node $i$ is displayed as follows.

$$
\underset{z_{f i}, l_{i}, g_{i}}{\operatorname{maximize}} \quad p_{i} \sum_{f} z_{f i}-\int_{l_{i}}^{K_{i}} B_{i}^{\prime}(x) d x-C_{i}^{g}\left(g_{i}\right)
$$

subject to

$$
\begin{array}{ll}
\sum_{f} z_{f i}+l_{i}-K_{i}-g_{i} \leq 0, & \\
g_{i} \leq G_{i} & \left(\kappa_{i}\right), \\
-\sum_{f} z_{f i}-l_{i} \leq 0 & \left(\mu_{i}\right), \\
l_{i}, g_{i} \geq 0 . &
\end{array}
$$

The three terms in the objective function of (2), in order, correspond to revenue (+) or cost (-) from transactions with the wholesale market, foregone benefit (if $K_{i}>l_{i}$ ) or incremental benefit (if $l_{i}>K_{i}$ ) of consuming power, and generation costs incurred from backup generation, respectively. Three constraints are associated with the prosumers' problem. (2b) states that the sum of renewable output $K_{i}$ and self generation $g_{i}$ net of sales to the wholesale market or $\sum_{f} z_{f i}$ has to be greater than the quantity consumed $l_{i}$. (2c) limits the output $g_{i}$ by its capacity $G_{i}$, and (2d) indicates that prosumers cannot purchase more than its demand.

While a prosumer only participates in the wholesale market indirectly through bilateral contracts rather than, say directly submitting bids into the market, one can assume that it acquires "strategic" knowledge through its repeated observations of power price clearance processes of the wholesale market.

$\underset{x_{f i h}, y_{i}, d_{i}}{\operatorname{maximize}} \sum_{i}\left(P_{i}^{0} d_{i}-\frac{P_{i}^{0}}{2 Q_{i}^{0}} d_{i}^{2}\right)-\sum_{f i h} C_{f i h}\left(x_{f i h}\right)$

subject to

$$
\begin{array}{lll}
x_{f i h} \leq \quad\left(\beta_{f i h}\right), & \\
-x_{f i h} \leq & 0 & \left(\varepsilon_{f i h}\right), \\
\sum_{i} P T D F_{k i} y_{i}-T_{k} \leq & 0 & \left(\lambda_{k}^{+}\right), \\
-\sum_{i} P T D F_{k i} y_{i}-T_{k} \leq & 0 & \left(\lambda_{k}^{-}\right),
\end{array}
$$




$$
\begin{array}{lrr}
d_{i}-\sum_{f, h \in H_{f i}} x_{f i h}-\sum_{f} z_{f i}=y_{i} & \left(\eta_{i}\right) \\
\sum_{i} y_{i}= & 0 & (\theta) \\
-d_{i} \leq & 0 & \left(\xi_{i}\right)
\end{array}
$$

The lower level problem is the social surplus maximization problem faced by the grid operator and is formulated in (3). Constraint (3f) is the nodal balance with prosumer's output $\left(z_{f i}\right)$ embedded. The balance between supply and demand is implied in $(3 \mathrm{~g})$.

\subsubsection{Lower-Level Problem}

$\underset{\Omega \cup \Delta}{\operatorname{maximize}} \sum_{i} \eta_{i}\left(\sum_{f} z_{f i}\right)-\int_{l_{i}}^{K_{i}} B_{i}^{\prime}(x) d x-C_{i}^{g}\left(g_{i}\right)$

subject to

$\sum_{f}\left(z_{f i}\right)+l_{i}-K_{i}-g_{i} \leq 0$

$g_{i} \leq G_{i}$

$-C_{f i h}^{\prime}\left(x_{f i h}\right)-\beta_{f i h}+\varepsilon_{f i h}+\eta_{i}=0 \quad f, i, h$,

$P_{i}^{0}-\frac{P_{i}^{0}}{Q_{i}^{0}} d_{i}-\eta_{i}+\xi_{i}=\quad 0 \quad \forall i \in I$

$-\sum_{k}\left(\lambda_{k}^{+}-\lambda_{k}^{-}\right) P T D F_{k i}+\eta_{i}-\theta=0 \quad \forall i \in I$,

$0 \leq \beta_{f i h} \perp\left(x_{f i h}-X_{f i h}\right) \quad \leq 0$,

$d_{i}-\sum_{f, h \in H_{f i}} x_{f i h}-\sum_{f} z_{f i}=\quad y_{i}$

$0 \leq \lambda_{k}^{+} \perp \sum_{i} P T D F_{k i} y_{i}-T_{k} \quad \leq 0$,

$0 \leq \lambda_{k}^{-} \perp-\sum_{i} P T D F_{k i} y_{i}-T_{k} \leq 0$,

$\sum_{i} y_{i}=0$

$0 \leq \varepsilon_{f i h} \perp x_{f i h} \quad \geq 0$,

$0 \leq \xi_{i} \perp d_{i}$

$\geq 0$,

$l_{i}, g_{i} \geq 0$.

So, constraint (3g) implicitly incorporates the supply and demand balance. Notice that the social surplus maximization problem does not include sales $\left(s_{f i}\right)$ of each generation firm as a decision variable but rather decides on their output $\left(x_{f i h}\right)$ and the bilateral transaction with the prosumer $\left(z_{f i}\right)$. However, once $x_{f i h}$ and $z_{f i}$ are decided by the solution of the problem, the sales balance for the generation firm holds automatically and would be consistent with (3f) and $(3 \mathrm{~g})$. Furthermore, the grid operator welfare/surplus maximization problem does not directly involve the grid operator revenues. However, we calculate the revenues of the grid operator by calculating the flows and recovering the transmission charge $\left(w_{i}\right)$, which indicates the price $(\$ / \mathrm{MW})$ charged by the grid owner to move power from hub to node $i$. This transmission charge is equal to the difference between the hub price and price at node $i$ which are readily calculated in the model [14].

\subsection{MPEC Formulation}

Following the formal definition of MPEC in Section 2.1, we introduce the leader-follower formulation of the Stackelberg in the electricity market.

Here, the upper-level problem is the prosumer's benefit maximization, and the lower-level problem is the equilibrium (or complementarity) conditions in the market derived from the grid operator's social surplus maximization problem. The MPEC formulation is given in (4).

The objective function is the benefit of the prosumer with prices derived from the dual variable associated with the nodal balance constraint in the lower-level problem. Constraints (4b)-(4d) indicate the operational constraints of the prosumer. Constraints $(4 \mathrm{e})-(4 \mathrm{~m})$ include the optimality conditions of the lower level problem that, together with the nodal balance constraint, form the complementarity problem characterizing the equilibrium of the market.

The problem is subject to two forms of non-convexities in (4): the bilinear term $\left(\eta_{i} \sum_{f} z_{f i}\right)$ in the objective function and the complementarity conditions defining the constraint set. We overcome these by, first, replacing the complementarity conditions with disjunctive constraints [22]. We then bypass the bilinear terms by applying the Wolfe's duality and recast the problem into a mixed integer quadratic program (MIQP).

\section{Numerical Example}

\subsection{Assumptions}

The model is applied to the IEEE Reliability Test System (aggregator) [23]. The topology of the system consists of 24 buses, 38 transmission lines, 
and 17 constant-power loads with a total of 2,850 MW. We aggregate 32 generators into 13 generators by combining those with the same marginal cost and located at the same node. Six generation units, however, are excluded from the dataset since they are hydro power units, which operate at their maximum output of $50 \mathrm{MW}$ [24]. Because the wholesale market is assumed to be perfectly competitive, we assume that all the generators are owned by a single firm. In order to be able to analyze the impact of transmission congestion, the capacity of line 7 in the test case is reduced to $150 \mathrm{MW}$. The marginal cost of generation is represented by a quadratic function parameterized by $C_{0}$ and $C_{1}$ as the coefficient for the linear and quadratic term, respectively. While using the IEEE RTS 24-bus system might be overly simplified the real-world power system, it allows us to focus on the impacts of strategic prosumers on the market.

Furthermore, the prosumer, or the leader, is assumed to be located at node 1 with the same preferences and quantity demanded of power consumption as consumers located in that node. That is, both the prosumer and the consumers in node 1 are assumed to have the same demand function. The prosumer owns a renewable generating unit that produces a variant amount of power (contingent on available natural resources) and a dispatchable unit as a backup option.

The RTS 24-Bus case is first formulated as a least-cost minimization problem and solved with fixed nodal load in order to get dual variables associated with load constraints. The dual variables together with an assumed price elasticity of -0.2 is then used to calculate $P_{i}^{0}$ and $Q_{i}^{0}$. The magnitude of price elasticity of demand is comparable with what has been reported in [25].

We examine three scenarios, varied by the levels of renewable output from the units owned by the prosumers. More specifically, renewables output $K_{1}$ is assumed to have three levels: 25, 50, and $120 \mathrm{MW}$. The levels might seem too big for an individual prosumer to amass. One can consider the entity as a super prosumer or an aggregator who represents a plethora of small prosumers. Also, These levels are chosen carefully in order to illustrate market outcomes.

\subsection{Results and Discussion}

Table 1 summarizes market outcomes when the prosumer is formulated as a Stackelberg leader for three scenarios: 25,50 , and $120 \mathrm{MW}$ of renewable generation. As indicated in the first row of Table 1, prosumer's changes from purchase (-) to sale (+) with increased levels of renewable output. For cases with 25 and $50 \mathrm{MW}$, the prosumer buys $59.50 \mathrm{MWh}$ and 39.61
Table 1. Results of the MPEC Model

\begin{tabular}{l|rrr}
\hline Variables \Scenarios & (a) & $(\mathrm{b})$ & $(\mathrm{c})$ \\
\hline Renewable output [MW] & 25 & 50 & 120 \\
\hline Prosumer's sale(+)/purchase(-) [MWh] & -59.50 & -39.61 & 18.61 \\
Prosumer's load [MWh] & 99.48 & 101.40 & 105.83 \\
Prosumer's generation [MWh] & 14.97 & 11.79 & 4.44 \\
Marginal cost of backup [\$/MWh] & 49.97 & 46.79 & 39.44 \\
Prosumer's surplus [\$K] & 9.85 & 11.06 & 14.05 \\
\hline Total power demand [MWh] & $2,848.55$ & $2,852.16$ & $2,858.69$ \\
Total power production [MWh] & $2,908.05$ & $2,891.77$ & $2,840.08$ \\
Power price in node 1 [\$/MWh] & 45.21 & 43.62 & 40.35 \\
Sale-weighted power price [\$/MWh] & 35.48 & 35.38 & 35.17 \\
\hline Grid operator's revenue [\$K] & 9.67 & 8.15 & 5.14 \\
Producers' surplus [\$K] & 41.93 & 42.89 & 44.98 \\
Consumers' surplus [\$K] & 255.89 & 256.36 & 257.25 \\
Social Surplus [\$K] & 307.49 & 307.40 & 307.37 \\
\hline
\end{tabular}

MWh, respectively. Thus, it is in a short position. As expected, the quantity of the purchases decreases as the prosumer's renewable output grows. In the third column, where the renewable output is equal to 120 $\mathrm{MW}$, the prosumer lies in a long position in equilibrium and sells (positive quantity) 18.61 MWh to the power market.

Prosumer's quantity demanded, or load, indicated in the second row of Table 1. Similarly, it increases as the prosumer has more renewable generation resources available and is equal to $99.48,101.40$, and 105.83 MWh for columns (a), (b), and (c), respectively. Having more zero marginal cost renewable by the prosumer (moving from (a) to (c)) implicitly shifts the supply curve to the right, leading to an increase in electricity consumption. This in turn decreases prosumer's generation from the dispatchable unit where the prosumer requires less generation from the backup unit given higher levels of renewable capacity. This effectively reduces the marginal cost of the backup unit as more renewable resources become available as shown in Table 1 where the marginal cost of the backup unit decreases from $49.97 \$ / M W h$ in column (a) to 39.44 $\$$ MWh in column (c).

Turning to prosumer's profit, the prosumer benefits from having more renewable generation output. This is because having more zero marginal cost resources, the prosumer is able to rely less on backup unit (lower operating cost), sell more to (buying less from) the market, thereby leading to higher benefit. As seen in Table 1 (also in Figure 2), the prosumer surplus follows an increasing trend of $9.85,11.06$, and $14.05(\$ \mathrm{~K})$ for 25,50 , and $120 \mathrm{MW}$ of renewable output, respectively.

The power price in node 1 , where the prosumer resides, is directly affected by the available renewables: it drops with an increasing amount of renewables. For example, when the renewable capacity is $25 \mathrm{MW}$, power price at node 1 is $45.21 \$ / \mathrm{MWh}$, which is reduced to 43.62 and $40.35 \$ / \mathrm{MWh}$ if the renewable generation capacity is 50 and $120 \mathrm{MW}$, respectively. 
We can also observe that, over the entire grid, the sale-weighted average price of electricity is reduced as the prosumer possesses more renewable generation output. As indicated in Table 1, the sale-weighted power price is reduced from $35.48 \$ / \mathrm{MWh}$ in column (a) to 35.38 \$/MWh and 35.17 \$/MWh in columns (b) and (c), respectively.

As the sale-weighted power price decreases with higher levels of renewable output owned by the prosumer, the total demand in the market increases when more zero marginal cost resource is available. As shown in Table 1, the total power demanded follows an increasing trend of 2,848.55 to 2,852.16 and 2,858.69 MWh from columns (a) to (c) as the prosumer's renewable output increases. Similarly, with more renewables, the prosumer engages in less purchase from the market (if in the short position) or sells more to the market (if in the long position), thereby mitigating the need of generation from conventional producers, hence reducing the total power generation from conventional units. This is shown in Table 1, where total power production is decreased from 2,908.05 MWh in the case of $25 \mathrm{MW}$ renewable capacity to 2,891.77 $\mathrm{MWh}$ and 2,840.08 MWh when the renewable capacity is 50 and $120 \mathrm{MW}$, respectively.

Since less generation from conventional units means that the the grid operator would need to move less power around in the grid, the grid operator's revenue decreases with higher levels of renewable capacity for the prosumer. Table 1 illustrates the fact that the grid operator's revenue $(\$ \mathrm{k})$ is reduced from 9.67 in the case of $25 \mathrm{MW}$ renewable capacity to 8.15 and 5.14 when the renewable capacity is 50 and $120 \mathrm{MW}$, respectively.

The producer's surplus increases with amount of renewable available to the prosumer. Although the right-shift of supply curve (due to growing renewable) effectively lowers the equilibirum prices, the profit earned by producers is more than made-up by power sales, leading to an increase in profits. This is illustrated in Table 1 where the producer surplus (in \$k) increases from 41.93 to 42.89 and 44.98 in columns (a), (b), and (c), respectively.

With increased consumption (demand) and lower prices, the consumers are poised to gain from increased levels of prosumer's renewable output. Indeed, this is what emerges in Table 1 where the consumer surplus (in $\mathrm{k} \$$ ) increases monotonically from 255.89 to 256.36 and 257.25 in columns (a), (b), and (c). The grid operator's revenue loss from increased levels of prosumer's renewable capacity is not sufficiently compensated by the growth in prosumer, consumers, and producers' surplus, and hence the social surplus decreases as the prosumer possesses more renewable
Table 2. Prosumer Surplus [\$K] Following Different Strategies

\begin{tabular}{l|rrr}
\hline Strategy $\backslash$ Scenarios & (a) & (b) & (c) \\
\hline Renewable output $[\mathrm{MW}]$ & 25 & 50 & 120 \\
\hline Stackelberg & 9.85 & 11.06 & 14.05 \\
Price-Taker & 9.83 & 11.05 & 14.05 \\
Cournot & 9.23 & 10.78 & 13.99 \\
\hline
\end{tabular}

generation capacity. This is shown in Table 1 where the social surplus (in \$k) is 307.49, 307.40, and 307.37 for 25,50 , and $120 \mathrm{MW}$ of renewable capacity, respectively.

Table 2 (also in Figure 2) illustrates the prosumer surplus under different strategies in the power market with different levels of renewable generation output. In column (a), where the prosumer's zero marginal cost resource is equal to $25 \mathrm{MW}$, the solution indicates that Stackelberg strategy yields a higher level of profit for the prosumer, although indiscernible from that of price-taker in Figure 2. Namely, the leader-follower equilibrium of the game induced in the power market would yield a surplus of $\$ 9.85 \mathrm{~K}$ which is higher than that of a price-taker strategy with a profit of $\$ 9.83 \mathrm{~K}$ and $\$ 9.23 \mathrm{~K}$ for the case of Cournot strategy; a similar pattern can be seen in column (b) and (c). When the prosumer renewable generation capacity is $50 \mathrm{MW}$, the surplus following a Stackelberg strategy is $\$ 11.06 \mathrm{~K}$ which is higher than $\$ 11.05 \mathrm{~K}$ and $\$ 10.78 \mathrm{~K}$ for the cases of price-taker and Cournot strategies, respectively. In column (c), where the renewable generation capacity is $120 \mathrm{MW}$, we observe that Stackelberg and perfect competition equilibria yield an equal prosumer surplus of $\$ 14.05 \mathrm{~K}$, which is higher than the $\$ 13.99 \mathrm{~K}$ surplus for the Cournot case. This indicates that as the prosumer's renewable generation generation increases, we begin to see the closing of the gap in prosumer surplus following different strategies. In other words, the prosumer plays a more pivotal role in the market, and hence in regard to its own profits, with lower levels of renewable capacity and as the zero marginal cost resources are expanded, the overwhelming increase on the supply-side undermines the choice of strategy. To further analyze the impact of prosumer presence in power markets, we compare the outcomes of a Stackelberg formulation to the cases of perfect competition and Cournot with various levels of $K_{i}$ ranging from $25 \mathrm{MW}-120 \mathrm{MW}$.

First, we look at the prosumer's output or interaction in the market. Figure 1 plots the prosumer's sale (+) or purchase (-) in perfect competition (PC), Cournot and Stackelberg cases against the zero-marginal cost renewable's output in x-axis from 25 to $120 \mathrm{MW}$. The horizontal dotted line crossing zero on the y-axis 
Figure 1. Prosumer's sales $(+)$ or purchase $(-)$ under various levels of renewables

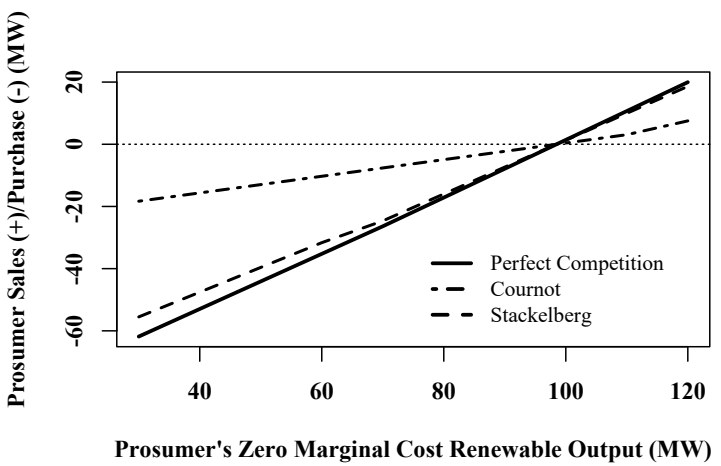

Figure 2. Prosumer's Surplus under various levels of renewable capacity

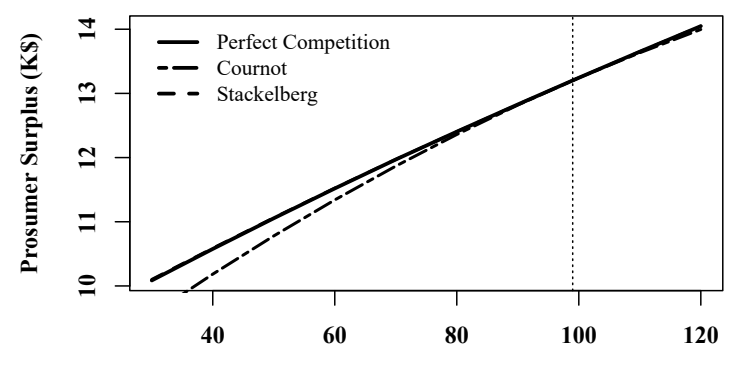

Prosumer's Zero Marginal Cost Renewable Output (MW)

indicates the island mode where the prosumer is isolated from the grid. The prosumer surplus or benefit is also plotted in Figure 2 for different levels of renewable capacity. Despite the lines overlapping, in fact, the prosumer surplus in the Stackelberg equilibrium, for any level of renewable capacity, is higher than that of perfect competition and Cournot as in Table 2.

Figure 1 also indicates that, in the short position when the prosumer purchases from the grid, for any level of renewable output, the quantity purchased under the Stackelberg strategy is sandwiched between PC and Cournot. The same phenomenon is observed for the long position when the prosumer sells power to the grid. In other words, the prosumer following a Stackelberg strategy would reduce purchases (sales) in the short (long) position compared to the case of perfect competition but increase purchases (sales) in the short (long) position compared to the Cournot case. In [19],
Figure 3. Power price in the prosumer's node (\#1) against various levels of renewables

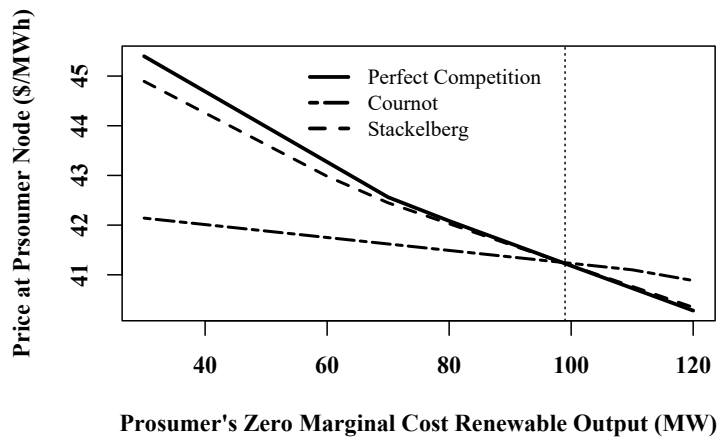

we show that the prosumer is always better off when behaving as a price-taker. This is consistent with our observations in Figure 1 as the prosumer's sell/purchase in the Stackelberg is distanced from the Cournot case and closer to the plot for the perfect competition.

Figure 3 shows the changes in the price at the prosumer's node given various levels of renewable generation output. Consistent with the finding in Table 1 , the price monotonically decreases as the prosumer possesses larger amounts of renewable output, thereby increasing supply and hence reducing the price. This pattern holds also for all three strategies. The vertical dotted line close to the $100 \mathrm{MW}$ on the horizontal axis indicates the island mode where the prosumer is "isolated" from the main grid. To the left of the vertical dotted line indicates that the prosumer experiences a short position in equilibrium where the prosumer purchases power from the market, and to the right is the long position where the prosumer sells power to the market. Again, we observe that the outcomes (in this case, the price) in the Stackelberg formulation is sandwiched between those of the perfect competition and Cournot cases regardless of the prosumer's position (short or long) in the market. This is consistent with what we observed in Figure 1. In the long position, where the prosumer output (supply) is less than the price-taker and more than the Cournot case, the price in the Stackelberg equilibrium would consequently be higher than the price-taker and lower than the Cournot case as shown in Figure 3. The same argument applies for the short position.

The joint impact of prosumer's behavior and amount of renewables is also propagated to the rest of the grid. Figure 4 gives results of the wholesale market's sale-weighted price. Similar to the findings in Figure 3, 
Figure 4. Average sale-weighted power price against various levels of renewables

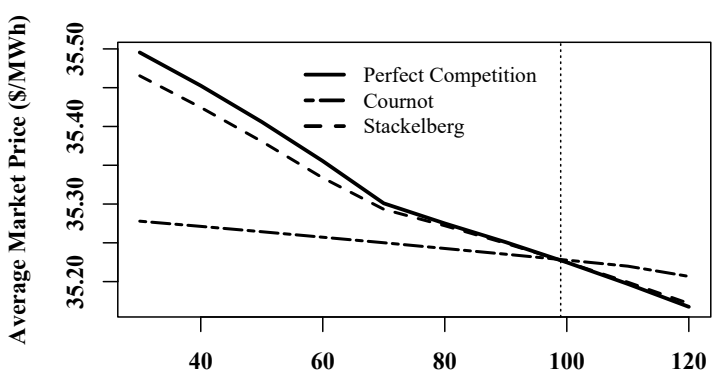

Prosumer's Zero Marginal Cost Renewable Output (MW)

Figure 5. Producer's surplus against various levels of renewables

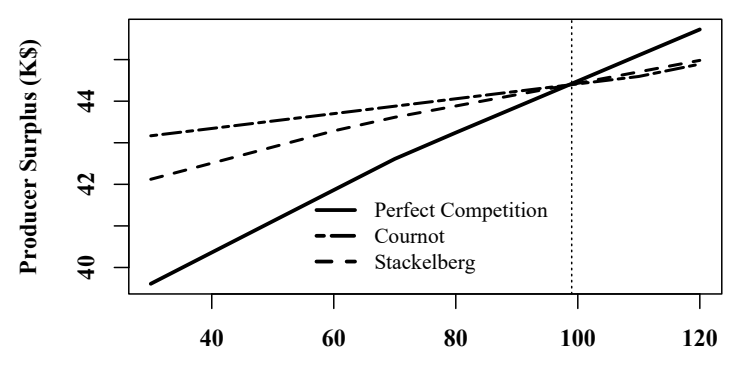

Prosumer's Zero Marginal Cost Renewable Output (MW)

it shows that the average market price in the Stackelberg equilibrium, regardless of the amount of renewables, is in-between that of the perfect competition and Cournot cases. The sale-weighted prices also decline with an increasing amount of renewables possessed by the prosumer.

Economic rent distributed among entities in the power market is displayed in Figures 5-7. Figure 5 illustrates the producer's surplus. Again, the horizontal axis is the prosumer's renewable output; the vertical axis indicates the producer surplus in $[\mathrm{k} \$]$ and the vertical dotted line indicates the island mode. The outcome of the Stackelberg equilibrium lies in between those of perfect competition and Cournot cases. Since in the Stackelberg equilibrium, the prosumer output is higher (lower) than the perfect competition (Cournot) case, the generation cost (and hence the producer surplus) would also be higher (lower) than the perfect competition
Figure 6. Consumer's surplus against various levels of renewables

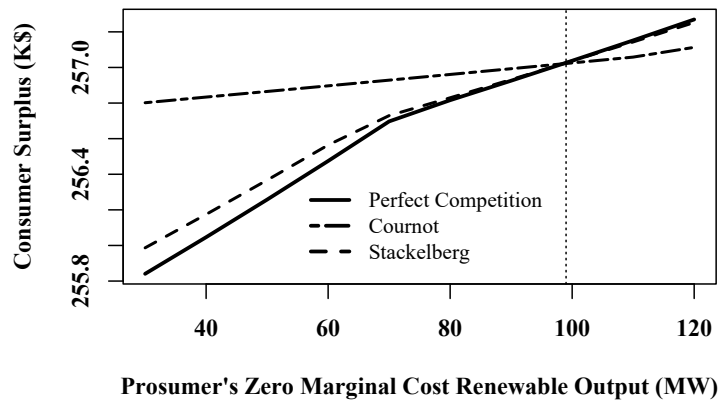

(Cournot) as shown in Figure 5, independent of the level of prosumer renewable output.

Figure 6 illustrates the consumer's surplus resulting from different strategies by the prosumer in the power market given various levels of prosumer renewable generation output. As discussed in Table 1, the consumer's surplus monotonically increases with higher levels of prosumer renewable output regardless of the underlying strategy as more renewable output is equivalent to more supply-side zero marginal cost resources. We observe that the consumer's surplus in the Stackelberg equilibrium is sandwiched between that of perfect competition and Cournot equilibria. In the short position, when the prosumer, as the leader, decreases (increases) power price compared to the perfect competition (Cournot) cases, the consumer's surplus is consequently increased (reduced) compared to the perfect competition (Cournot) case as shown in Figure 6. Similarly, in the long position, once the prosumer increases (decreases) power prices in the Stackelberg equilibrium compared to those of perfect competition (Cournot), the consumer surplus consequently increases (decreases) above (below) the levels of Cournot (perfect competition).

Figure 7 suggests that the grid operator's revenues are monotonically decreasing with the amounts of prosumer's renewable output as higher levels of zero marginal cost resources would mitigate the need for generation, thereby utilizing less transmission, lowering the grid operator's revenues. Interestingly, for the grid operator's revenues as well, we are able to observe the outcome of the Stackelberg equilibrium lying in-between that of Cournot and perfect competition. In the short position, focusing on the Stackelberg equilibrium, the prosumer purchase is less (more) than 
Figure 7. The grid operator's revenue against renewable outputs

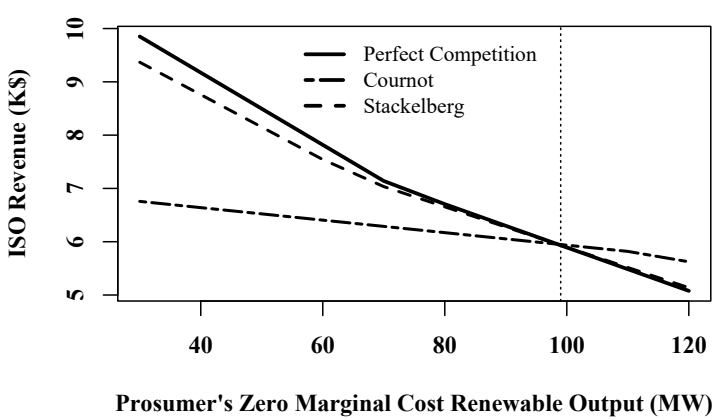

Figure 8. Social surplus against various levels of renewables

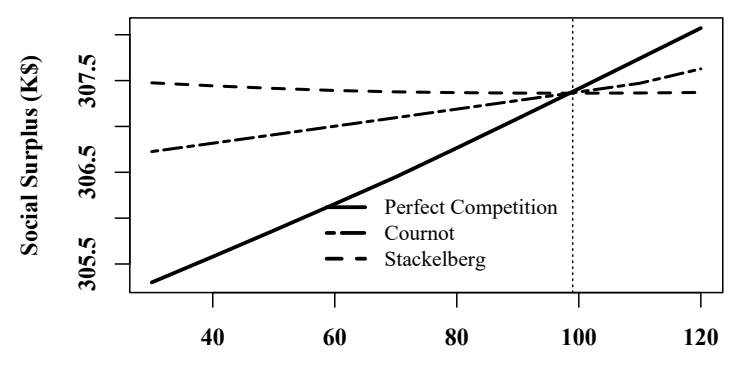

Prosumer's Zero Marginal Cost Renewable Output (MW)

that of Cournot (perfect competition), which in turn necessitates less (more) power generation (and power flow, hence the grid operator's revenues) compared to the cases of perfect competition (Cournot). In the long position, similarly, the prosumer's sale to the market in the Stackelberg equilibrium is more (less) than that of Cournot (perfect competition), which leads to less (more) need for power generation from conventional producers and hence less (more) power to be moved around the grid that leads to less (more) grid operator's revenues compared to Cournot (perfect competition) case.

Figure 8 displays the wholesale's social surplus (exclusive of prosumer's profit) under three cases over various renewable output. Two observations emerge. First, the social surplus monotonically increases with levels of renewable output for Cournot and perfect competition cases. However, somehow surprisingly, under the Stackelberg case, it actually decreases with the increased level of renewable output. A further comparison among different cases suggests that this is mainly because the increases in the consumers' and producers' surplus in Figures 6 and 5 is more than offset by the drop of the grid operator's revenue in Figure 7, leading to a decline in the social surplus. Second, the prosumer's strategy yields different order of the social surplus among cases in the short and long position. In particular, unlike cases in previous figures, the social surplus is no longer sandwiched between the price-taker and Cournot cases, thereby deviating from the conventional wisdom. The fact that the prosumer is "not" part of the wholesale market might be the source of the difference.

\section{Conclusions}

Prosumers, an emerging entity for enhancing sustainability and resilience of the power sector, are expected to play an increasingly important role in the electricity market. The dual nature of the prosumer, i.e., their ability of concurrent generation and consumption, while introducing new opportunities, also poses significant challenges in the energy sector. The present paper extends the existing work on the role of prosumers in the electricity market by formulating the prosumers as a leader within a Stackelberg framework. We model the prosumer as an agent, possessing limited renewable as well as dispatchable generation capacity, maximizing its net benefit by deciding on the level of trade (sale or purchase of power) with the wholesale market, level of forgone consumption, and level of generation from the dispatchable unit.

We investigate the market outcomes in a Stackelberg setting and compare the results to those where the prosumer is designated as a price-taker and a Cournot entity in the market. The results indicate that market outcomes are affected by the prosumer's strategy, i.e., price-taking, Cournot, and Stackleberg, as well as the amount of its possessed renewables. Possessing relatively lower (higher) renewable, the prosumer behaves as consumers (producers) and purchase from (sell to) the main grid. The outcomes of the Stackelberg competition lie between those of Cournot (least competitive) and perfect competition (most competitive) scenarios. For instance, price-taking prosumers purchase more power from the main grid, followed by Stackelberg and Cournot (see Figure 1). Under the relatively larger amount of renewable, the outcomes asymptotically approach that of perfect competition. This observation is consistently observed in Figures 1-4, and 6. However, although the impact of the strategic 
prosumer on each of the market participants in the Stackelberg case was in between perfect competition and imperfect competition (Cournot), its impact on the social surplus is ambiguous. More specifically, in the short position, the prosumer in the Stackelberg equilibrium increases the social surplus, higher than perfect competition and Cournot. On the other hand, in the long position, the prsumer's strategy leads to a decline in social surplus, lowest compared to perfect competition and Cournot cases.

\section{Acknowledgment}

This work was supported by a grant from NSF under the contract CMMI-1832683.

\section{References}

[1] F. Rahimi and A. Ipakchi, "Demand response as a market resource under the smart grid paradigm," IEEE Transactions on smart grid, vol. 1, no. 1, pp. 82-88, 2010.

[2] C. J. Day, B. F. Hobbs, and J.-S. Pang, "Oligopolistic competition in power networks: A conjectured supply function approach," IEEE Transactions on Power Systems, vol. 17, pp. 597-607, Aug. 2002.

[3] B. E. Hobbs, "Linear complementarity models of Nash-Cournot competition in bilateral and POOLCO power markets," IEEE Transactions on Power Systems, vol. 16, pp. 194-202, May 2001.

[4] L. Gkatzikis, I. Koutsopoulos, and T. Salonidis, "The role of aggregators in smart grid demand response markets," IEEE Journal on Selected Areas in Communications, vol. 31, no. 7, pp. 1247-1257, 2013.

[5] A. Papavasiliou and S. S. Oren, "Large-scale integration of deferrable demand and renewable energy sources," IEEE Transactions on Power Systems, vol. 29, no. 1, pp. 489-499, 2013.

[6] S. Bose, C. Wu, Y. Xu, A. Wierman, and H. Mohsenian-Rad, "A unifying market power measure for deregulated transmission-constrained electricity markets," IEEE Transactions on Power Systems, vol. 30, no. 5, pp. 2338-2348, 2014.

[7] Y. Yu, B. Zhang, and R. Rajagopal, "Do wind power producers have market power and exercise it?," in 2014 IEEE PES General Meeting - Conference \& Exposition, pp. 1-5, IEEE, 2014.

[8] C. Chen, S. Kishore, Z. Wang, M. Alizadeh, and A. Scaglione, "How will demand response aggregators affect electricity markets? \#x2014; A Cournot game analysis," in 2012 5th International Symposium on Communications, Control and Signal Processing, pp. 1-6, May 2012.

[9] N. A. Ruhi, K. Dvijotham, N. Chen, and A. Wierman, "Opportunities for Price Manipulation by Aggregators in Electricity Markets," IEEE Transactions on Smart Grid, pp. 1-1, 2017.

[10] K. Alshehri, M. Ndrio, and S. B. T. Basar, "The impact ofaaggregating distributed energy resources on electricity market efficiency," in Annual Conference on Information Sciences and Systems (CISS), 2019.
[11] Y. Chen, B. F. Hobbs, S. Leyffer, and T. S. Munson, "Leader-follower equilibria for electric power and no $_{x}$ allowances markets," Computational Management Science, vol. 3, no. 4, pp. 307-330, 2006.

[12] R. Entriken and S. Wan, "Agent-based simulation of an automatic mitigation procedure," in Proceedings of the 38th Annual Hawaii International Conference on System Sciences, pp. 92c-92c, IEEE, 2005.

[13] R. Entriken and S. Wan, "Pushing capacity payments forward: agent-based simulation of an available capacity market," tech. rep., EPRI Technical Report (Available at www. epriweb. com/public ..., 2003.

[14] B. F. Hobbs, C. B. Metzler, and J.-S. Pang, "Strategic gaming analysis for electric power systems: An mpec approach," IEEE transactions on power systems, vol. 15, no. 2, pp. 638-645, 2000.

[15] X. Hu, D. Ralph, E. K. Ralph, P. Bardsley, M. C. Ferris, et al., "Electricity generation with looped transmission networks: Bidding to an iso," Research Paper, vol. 16, 2004.

[16] G. Pepermans and B. Willems, "Ramsey pricing in a congested network with market power in generation: A numerical illustration for belgium," 2005.

[17] S. A. Gabriel and F. U. Leuthold, "Solving discretely-constrained mpec problems with applications in electric power markets," Energy Economics, vol. 32, no. 1, pp. 3-14, 2010.

[18] S. Siddiqui and S. A. Gabriel, "An sos1-based approach for solving mpecs with a natural gas market application," Networks and Spatial Economics, vol. 13, no. 2, pp. 205-227, 2013.

[19] S. Ramyar, A. Liu, and Y. Chen, "A power market model in presence of strategic prosumers." unpublished, 2019.

[20] Z.-Q. Luo, J.-S. Pang, and D. Ralph, Mathematical programs with equilibrium constraints. Cambridge University Press, 1996.

[21] L. Kristov, "The bottom-up (r) evolution of the electric power system: The pathway to the integrated-decentralized system," IEEE Power and Energy Magazine, vol. 17, no. 2, pp. 42-49, 2019.

[22] S. A. Gabriel, A. J. Conejo, J. D. Fuller, B. F. Hobbs, and C. Ruiz, "Optimality and complementarity," in Complementarity Modeling in Energy Markets, pp. 31-69, Springer, 2013.

[23] C. Grigg, P. Wong, P. Albrecht, R. Allan, M. Bhavaraju, R. Billinton, Q. Chen, C. Fong, S. Haddad, S. Kuruganty, W. Li, R. Mukerji, D. Patton, N. Rau, D. Reppen, A. Schneider, M. Shahidehpour, and C. Singh, "The IEEE Reliability Test System-1996. A report prepared by the Reliability Test System Task Force of the Application of Probability Methods Subcommittee," IEEE Transactions on Power Systems, vol. 14, no. 3, pp. 1010-1020, 1999.

[24] J. Wang, N. E. Redondo, and F. D. Galiana, "Demand-siderreserve offers in joint energy/reserve electricity markets," IEEE Transactions on Power Systems, vol. 18, no. 4, pp. 1300-1306, 2003.

[25] I. M. L. Azevedo, M. G. Morgan, and L. Lave, "Residential and regional electricity consumption in the us and eu: How much will higher prices reduce co2 emissions?," The Electricity Journal, vol. 24, no. 1, pp. 21-29, 2011. 